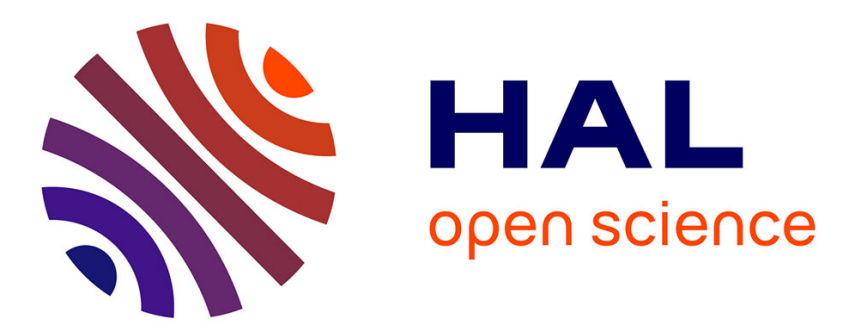

\title{
The Liquid Metal Ion Source as an Electrically Driven Vena Contracta, and some Comments on LMIS Stability
}

R. Forbes

\section{To cite this version:}

R. Forbes. The Liquid Metal Ion Source as an Electrically Driven Vena Contracta, and some Comments on LMIS Stability. Journal de Physique IV Proceedings, 1996, 06 (C5), pp.C5-43-C5-47. 10.1051/jp4:1996506 . jpa-00254385

\section{HAL Id: jpa-00254385 https://hal.science/jpa-00254385}

Submitted on 1 Jan 1996

HAL is a multi-disciplinary open access archive for the deposit and dissemination of scientific research documents, whether they are published or not. The documents may come from teaching and research institutions in France or abroad, or from public or private research centers.
L'archive ouverte pluridisciplinaire HAL, est destinée au dépôt et à la diffusion de documents scientifiques de niveau recherche, publiés ou non, émanant des établissements d'enseignement et de recherche français ou étrangers, des laboratoires publics ou privés. 


\title{
The Liquid Metal Ion Source as an Electrically Driven Vena Contracta, and some Comments on LMIS Stability
}

\author{
R.G. Forbes \\ University of Surrey, Department of Electronic and Electrical Engineering, Guildford, Surrey, GU2 5XH, \\ U.K.
}

\begin{abstract}
This paper discusses recent items of progress in understanding liquid-metal ion source (LMIS) behaviour, in the more general context of electrohydrodynamics. (1) Like the Taylor-Gilbert cone, the phenomenon of the vena contracta has been known for several hundred years. It is argued that the cusp-on-a-cone shape of an operating LMIS is a similar phenomenon, except that the forces acting are electrical rather than gravitational, and the pressure in the jet is negative rather than positive. (2) In discussing LMIS stability in engineering terms, mechanisms affecting the jet shape can be classified as giving negative feedback (stabilising), or positive feedback (destabilising). Effects associated with field evaporation and/or space charges give negative feedback, effects associated with pressure changes positive feedback. Attention is drawn to the arguments of Ganan-Calvo about the role of 'supercritical' flow as a stabilising factor against hydrodynamic disturbances. (3) Electrohydrodynamic spraying is superficially very similar to LMIS operation, and both sprayers and ion sources adopt a cusp-on-a-cone (or 'cone-jet') shape. But the accepted theoretical explanations of the driving mechanisms are different. Confirmation is offered that the LMIS story is the correct one for very highly conducting liquids, and that a difference in driving mechanism is plausible.
\end{abstract}

\section{INTRODUCTION}

A liquid metal ion source (LMIS) produces an optically bright, finely focused, ion beam. These sources have various practical applications $[1,2]$, including scanning ion beam instruments and integrated circuit mask repair. Over the years, steady progress has been made in establishing how the LMIS works $[2,3]$. Not surprisingly, the field-emission community (especially in the early days of the development of this topic in this community) has tended to concentrate more on the ion-emission aspects of these devices, notwithstanding the well-established fact that they intermittently emit liquid droplets. But slowly we have regained the perspective held by some of the original experimenters on these sources, for example [4], that, at least in some respects, they are specialised variants of electrohydrodynamic (EHD) sprayers.

Interest in the EHD aspects of LMIS behaviour has steadily increased, for example $[5,6]$, and there now seems growing scope for scientific interaction between those interested in LMIS theory and those interested in the theory of EHD spraying of non-metallic liquids. A special journal issue [7] some years ago provides a very useful overview of the general state of EHD spraying as a research topic.

This paper discusses several topics where small improvements have recently occurred in our basic physical understanding of hydrodynamic aspects of LMIS behaviour.

\section{THE TAYLOR-GILBERT CONE AS A VENA CONTRACTA}

A liquid under the influence of strong electric forces adopts a roughly conical shape. This effect was first reported by Gilbert [8] in 1600. The first person to produce an adequate theory was Taylor [9]. But Taylor notes that Gilbert reported them first, so I prefer to call these observed shapes 'Taylor-Gilbert cones', and reserve the name 'Taylor's mathematical cone' for Taylor's theoretical shape.

As is well known, Taylor's (hydrostatic) theory does not apply to the tip region of an operating liquid-metal ion source, which adopts a 'cusp-on-cone' shape that gets increasingly prominent as the applied voltage and 
emission current increase. Fig.1(a) below is redrawn from a transmission electron micrograph of an operating indium LMIS, taken recently by Praprotnik et al.[10]. EHD sprayers and electrospray. ion sources [11] also adopt this type of shape under many circumstances, and in this case are said to be operating 'in cone-jet mode'.

It is not surprising that a mathematical Taylor cone should develop into this shape when the voltage is increased, because this tends to develop a pressure gradient that causes liquid to move towards the apex [3]. But it has now been realised [3] that there is a well-known hydrodynamic phenomenon that offers a simple physical explanation of the cusp-on-a-cone shape.

Like the Taylor-Gilbert cone, the phenomenon of the vena contracta has been known for several hundred years. The typical situation in which the phenomenon occurs is when a jet of water emerges from a hole in the side of a cylinder. The cylinder needs to be full and the hole needs to be low in the side of the cylinder, so that the jet is driven by a pressure head of water. As the water jet emerges from the hole, the diameter of the jet decreases, so that close to the cylinder the water jet has a cusp-like shape, as shown schematically in Fig. 1(b) below. This is the vena contracta (see, for example, ref. [12]). It exists as a result of the pressure difference between the water in the cylinder, at the level of the hole, and atmospheric pressure outside the cylinder. This pressure difference causes the liquid to accelerate as it leaves the hole, and the equation of continuity requires the jet to respond by reducing its diameter as the water speed increases.

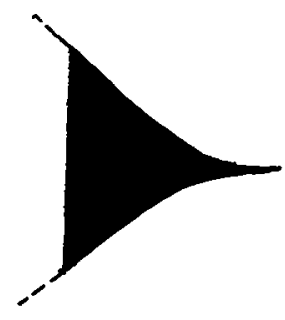

(a)

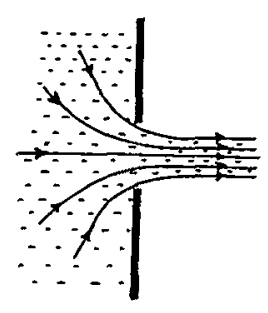

(b)

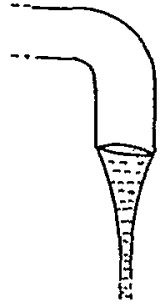

(c)

Figure 1. Three examples of a vena contracta: (a) a liquid-metal ion source; (b) water emerging from a hole in the side of a cylinder; (c) water emerging from a nearly-closed tap (faucet).

It can be argued that the cusp-on-a-cone shape of an operating liquid-metal ion source is basically the same phenomenon. The liquid metal begins by moving slowly upwards from the base of the Taylor-Gilbert cone. As the liquid approaches the the cusp region it accelerates, and (if simple calculations [13], based on a gallium ion current of $2 \mu \mathrm{A}$ are to be believed) has reached a speed of about $40 \mathrm{~m} / \mathrm{s}$ when it is in the columnar region of the jet. The acceleration occurs mainly in the base of the cusp. The difference between the LMIS situation and the classical hydrodynamic vena contracta is that the forces acting are electrical rather than gravitational, and that the pressure in the jet is negative rather than positive (i.e. the field 'sucks'; whereas the head of water 'pushes').

Another possible analogy is water emerging from a nearly closed tap, as shown schematically in Fig. 1(c).

In early theoretical discussions of liquid-metal ion sources, for example ref. [14], there was uncertainty over the shape of an operating needle-type LMIS, even though the cusp-on-a-cone shape of a capillary-type LMIS had already been observed by scanning electron microscopy [15]. If the LMIS is regarded as an electrically driven vena contracta, then its cusp-on-a-cone shape is - in terms of classical hydrodynamics exactly what one would expect. 


\section{AN ENGINEERING ORIENTED DISCUSSION OF LMIS STABILITY}

It is well established $[2,16]$ that liquid-metal ion sources emit microdroplets, and it is normally assumed that droplet emission is associated with the detachment of a large part of the liquid emitter, probably the whole of the jet and much of the cusp. Hornsey $[17,18]$ has also suggested processes that involve just the detachment of the emitting liquid apex, which I prefer to refer to as the formation of a 'nanodroplet'. Liquid globules can also detach themselves from the back end of the Taylor-Gilbert cone and/or from the supporting needle; for indium some spectacular HVTEM observations were recently reported by Praprotnik et al. [10].

This emission of liquid droplets should not be surprising, given that we are dealing with a liquid subject to Maxwell stresses, and given that electrohydrodynamic spraying is a well-established industrial technique. The surprising thing, perhaps, is that liquid-metal ion sources are as stable as they are. This section presents a qualitative analysis of possible reasons.

From analysis and modelling of the LMIS emission region, it emerges that the space-charge reduction in surface field is very marked (Swanson and Kingham [19] suggest that at the emitting liquid surface the Poisson field may be only one fifth of the Laplace field.) This provides a clue as to one reason for shortterm emitter stability. Suppose that, for some statistical reason, the ion emission rate decreases at the emitter tip. The space charge rapidly diminishes as the ions forming it move away, so the field at the liquid surface rapidly rises. The field evaporation rate-constant is very sensitive to the value of electric field, so ion emission rapidly resumes. This control process is sometimes called space-charge stabilisation.

The above, however, cannot be the whole of the story. For if the ion emission rate decreases, even momentarily, then the liquid jet will extend in length (because it's moving at very high speed!). This also will lead to an increase in field, but for geometrical rather than space-charge reasons; again, field evaporation will rapidly resume, and will presumably 'eat back' the jet to its original length. For want of a better name I call this inertial/geometrical stabilisation.

Analogous feedback mechanisms exist if, for some statistical reason, the ion emission rate suddenly increases: the enhanced space-charge, and/or the reduced length of the cusp, will reduce the surface field, and hence reduce the evaporation rate.

In engineering terms, all the above are mechanisms with intrinsic negative feedback. They all tend to encourage emitter stability.

In addition to the above, changes in the surface field will change the pressure difference between the body of the liquid cone and the tip of the liquid cusp, and hence the rate at which liquid flows to the cusp apex. With this mechanism, if the cusp increases in length, then (if we disregarded the stabilisation effects just discussed) the field at its apex would increase for geometrical reasons; this would increase the pressure difference between the tip of the cusp and the body of the liquid cone. This in turn would increase the flow rate; and the cusp would tend to grow further. In engineering terms, this mechanism has positive feedback: it is a destabilising mechanism, that I refer to as pressure-change destabilisation.

The author's belief is that pressure-change destabilisation effects of this type normally act more slowly than the stabilisation effects discussed above, and that this is a major reason why liquid-metal ion sources can provide relatively stable ion emission, at least for short periods of time.

A further issue is what might happen if the shape of the liquid cap changes temporarily from its steady-state shape. It is difficult to imagine that large hydrostatic pressure differences could exist stably in a volume of liquid about $3 \mathrm{~nm}$ across. So the steady-state shape of the cap must be such that the same difference exists everywhere between the magnitudes of the electrostatic and surface-tension stresses, although the stresses themselves may change with position on the surface as the local radius of curvature changes. If, as a result of some statistical fluctuation, the shape changes locally away from its steady-state shape, resulting in too high a local field, then expectation is that the field-evaporation rate will increase locally, and will locally "eat back' the cap to its local steady-state radius. This control mechanism is easy to understand, since a very similar mechanism operates in the low-temperature field evaporation of solids, where it produces the socalled field evaporation endform. For the LMIS we may call this process liquid endform stabilisation by field evaporation. 
Obviously, the rather basic discussion in this section has not dealt with the possible creation of fast-moving pressure waves in the liquid, and the possible effects of these [5]: it is these waves, the author suspects, that are responsible for (at least part of) the instability observed in real sources.

Another possible effect has been discussed by Ganan-Calvo [20], in the context of electrohydrodynamically driven jets. Ganan-Calvo argues that the motion of the liquid in the jet can become 'supercritical', i.e. the liquid speed can become sufficiently high that the liquid itself travels faster 'forwards' than a wave on its surface can travel 'backwards'. If the surface wave is a possible cause of instability, then in this situation of 'supercriticality' the jet may be protected against certain forms of instability.

Certainly we do not yet understand the details of the complicated interactions that may exist between the various processes mentioned above.

\section{ALTERNATIVE EXPLANATIONS FOR ELECTRICALLY DRIVEN JETS}

As already indicated, there seems to be a generic similarity between the mechanism of operation of a liquidmetal ion source and the operation of an electrohydrodynamic (EHD) sprayer in the so-called 'cone-jet' mode. However, explanations given of what drives the jet are significantly different. The LMIS story is that the jet is driven by a pressure difference between the apex of the liquid cusp and the base of the liquid cone: there is a negative pressure at the apex of the liquid cusp (typically -40 atmospheres for a gallium LMIS emitting at $2 \mu \mathrm{A}$, if simple calculations are to be believed [13]), due to a slight imbalance in the forces due to Maxwell stresses and surface tension forces; this is what drives the liquid motion.

In the case of the EHD sprayer, however, it is usually argued that the liquid motion is driven by 'lateral' electrical forces acting on the sides of the jet, as a result of a field gradient along the jet [21]. The argument is as follows: a field parallel to the surface of the jet acts on the surface charge, and pulls the charge towards the apex of the jet; the moving charge transfers momentum to the liquid, thereby accelerating it. (There is good evidence for validity of this driving mechanism in the case of some organic liquids, where fluid circulation can be seen clearly seen inside the liquid cone [22].)

These explanations are qualitatively different, and in principle both kinds of driving force may exist.

It is instructive to formulate a rough argument, for the liquid-metal ion source case, that enables the relative magnitudes of the driving forces to be estimated. Details will be presented elsewhere. The objective is to estimate the ratio $R$ of the total force $F_{\mathrm{p}}$ on the sides of the jet (parallel to its axis) to the total force $F_{\mathrm{n}}$ on the cap of the liquid emitter. It is possible to derive the following approximate formula:

$R=F_{\mathrm{p}} / F_{\mathrm{n}}=4 \times\left(\Delta V / E_{\mathrm{n}} r\right) \times\left(E_{\mathrm{s}} / E_{\mathrm{n}}\right)$,

where $r$ is the radius of the jet just below the liquid apex, $\Delta V$ is voltage drop along the jet, $E_{\mathrm{n}}$ is the field at the apex of the liquid cap, and $E_{s}$ is the average value of the component of field normal to the cylindrical surface of the jet, at this cylindrical surface, averaged along the length of the jet. The field ratio in the bracket needs to be estimated, and we assume here that it is not greater than $1 / 4$. This leads to:

$R \leq \Delta V / E_{\mathrm{n}} r$

For a gallium source, $E_{\mathrm{n}}=15 \mathrm{~V} / \mathrm{nm}$, and $r$ is typically equal to $1.5 \mathrm{~nm}$ or slightly more [13]. Field-ion energy-deficit measurements [23] allow us to put an upper limit on the voltage drop; I estimate this as not move than $2 \mathrm{~V}$, probably less. Hence we obtain $R \leq 0.09$.

This result confirms that for a liquid-metal ion source the main driving forces are at the apex of the emitter, as normally assumed. However, it easy to see that for a liquid of much lower electrical conductivity than a liquid metal (and organic liquids typically have conductivities lower than liquid metals by several or many orders of magnitude) the position could easily be reversed. In this case the main driving forces would be the lateral forces on the sides of the jet. So it seems entirely plausible that the driving forces for liquid-metal ion sources and electrostatic sprayers should be different. But this only makes it more intriguing to explain the observed commonalities of behaviour, in particular in respect of liquid shape. 


\section{REFERENCES}

[1] J Melngailis, J. Vac. Sci. Tech., B 5 (1987) 469.

[2] P D Prewett and G L R Mair, Focused Ion Beams from Liquid Metal Ion Sources . Research Studies Press, Taunton, UK (1991).

[3] Forbes R.G., Vacuum (to be published)

[4] Krohn V.E.., J. Appl. Phys. 45 (1974) 144.

[5] Vladimirov V.V., Badan V.E., Gorshkov V.N., Grechko L.G., Soloshenko A., J. Vac. Sc.. Tech. B 9 (1991) 2582.

[6] Mair G.L.R., J. Phys. D: Appl. Phys. 21 (1988) 1654.

[7] J. Aerosol Sci. 45 (1994) pp. 1005-1252.

[8] Gilbert W., De Magnete, Book 2, Chapter 2. London (1600). Translation by P F Mottelay published oy: Dover, New York (1958).

[9] Taylor G.]., Proc. Roy. Soc. Lond. A 280 (1964) 383.

[10] Praprotnik B., Driesel W., Dietzsch Ch., Niedrig N., Surface Sci. 314 (1994) 353.

[11] Diilcks Th., Röllgen F.W., Int. J. Mass Spectrom. Ion Processes 148 (1995) 123.

[12] Faber T.E., Fluid Dynamics for Physicists (Cambridge University Press, 1995).

[13] Forbes R.G. and Ljepojevic N.N., Surface Sci. 266 (1992) 170.

[14] Gomer R. Appl. Phys. 19 (1979) 365.

[15] Aitken K.I.., Field Emission Day - Proceedings of a Meeting held at ESTEC, Noordwijk, on 9 April 1976 (Report No. ESA SP 119), p 23. European Space Agency, Paris (1977).

[16] Barr D.L., Brown W.L., J. Vac. Sci. Tech. B 7 (1989) 1806.

[17] Hornsey F.I., PhD Thesis, University of Oxford (1989).

[18] Hornsey F.I., Jap. J. Appl. Phys. 30 (1991) 366.

[19] Swanson ]..W., Kingham D.R., Appl. Phys. A 41 (1986) 223.

[20] Ganan-Calvo A.M., J. Fluid Mech. (to be published).

[21] For examplle, Turnbull R.J., IEEE Trans. Industr. Applications 25 (1989) 699.

[22] Shtern V. and Barrero A., J. Aerosol Sci. 25 (1994) 1049.

[23] Swanson L.W., Nucl. Instr. Meth., 218 (1983) 347. 